\title{
Training AIBO like a Dog \\ - Preliminary results -
}

\author{
YAMADA Seiji \\ National Institute of Informatics \\ 2-1-2 Hitotsubashi, Chiyoda \\ Tokyo, 101-8430, Japan \\ seiji@nii.ac.jp
}

\author{
YAMAGUCHI Tomohiro \\ Nara National College of Technology \\ 22 Yata-cho, Yamato-Koriyama \\ Nara, 639-1080, Japan \\ yamaguch@info.nara-k.ac.jp
}

\begin{abstract}
This paper describes a method to facilitate human adaptation to a pet robot. A pet robot learns which behavior it should execute when stimulus are given and a human user learns how to give commands to the robot through its various sensors. A pet robot utilizes a computational classical conditioning model for learning to interpret human's commands. We propose and implement such a mutual adaptation framework, and develop like-dog heuristics to facilitate the human's adaptation to a pet robot AIBO. Finally we evaluate the heuristics through preliminary experiments.
\end{abstract}

\section{Introduction}

Home robots like AIBO[2], Roomba are gradually spreading for entertainment and housework as progression of robotics technology. When home robots come in house, people inevitably have interaction with them and design of such interaction is becoming important research issues in robotics. Thus human-robot interaction, especially the interaction between home robots and naive people to it, is a very significant research target.

A human adapts to objects like machines, tools in nature. Thus when a human works and plays with a robot at home, he/she tries to adapt it by modeling it, predicting its behavior and teaching it something. In contrast, a robot should be designed to adapt to a human by learning the model and the action selection through interaction with him/her. Because a robot needs to be able to interpret user's commands and execute suitable actions depending on user's mental state. Hence mutual adaptation between a human and a pet robot, in which a human and a learning robot adapt each other, naturally occurs in humanrobot interaction. In such situation, efficiency of the mutual adaptation between a human and a learning robot significantly depends on design of interaction between them. In this literature, the interaction design includes not only design of modality, representation of information which a human and a robot communicate, but also design of robot's behaviors, learning algorithm on the human-robot interaction.

The objective of our study is to develop the methodology of designing human-robot interaction for facilitating mutual adaptation between a human and a robot. In this paper, first we design and implement a framework in which a human trains a pet robot AIBO to achieve a simple task. Classical conditioning is utilized to behavior learning of an AIBO. A human teacher trains an AIBO by simultaneously giving unconditioned stimulus and conditioned stimulus to it, and the AIBO gradually becomes able to execute suitable actions with only conditioned stimulus. For facilitating human's adaptation in this framework, we propose like-dog heuristics to design behavior of a pet robot, and actually describe behavior of an AIBO with the heuristics. Finally we conduct preliminary experiments to investigate the effectiveness of the likedog heuristics and show the results supporting our approach.

Human-robot interaction has thus far been studied between a human and mobile robots, humanoid robots. In particular, Ono and Imai proposed a cognitive model to describe how a human reads a robot mind and investigated its validity experimentally[9]. Though their work is interesting, it has no mutual learning of mind reading like our work.

We have researched mutual adaptation between a human and a life-like software agent[10]. We try to extend our mutual adaptation framework in which a human and a life-like agent adapt each other to humanrobot interaction,

Mutual adaptation between two humans is also interesting research issue. Komatsu et al. pointed out mutual adaptation between a human teacher and an human operator who cooperate each other to score in TV game[8]. Their study is closely related to our 
Table 1 Specification of AIBO

\begin{tabular}{|c|c|}
\hline Dimension & $180(\mathrm{~W}) \times 278(\mathrm{H}) \times 319(\mathrm{D}) \mathrm{mm}$ \\
Weight & Approx. $1.65 \mathrm{~kg}$ \\
CPU & MIPS R7000 $(576 \mathrm{MHz})$ \\
Sensors & Temperature Sensor \\
& Distance Sensor (head) \\
& Edge detection sensor (chest) \\
& Acceleration Sensor \\
& Tactile Sensor (head, back) \\
& Pressure Sensor (chin, paws (4)) \\
& CMOS camera 350,000 pixels \\
Actuators & Mouth: 1 dof \\
& Head: 3 dof \\
& Leg: 3 dof $\times 4$ legs \\
& Ear: 1 dof $\times 2$ \\
Tail: 2 dof \\
Wireless LAN
\end{tabular}

research, however they do not deal with mutual adaptation between a human and an agent like a pet robot.

Kaplan developed clicker training of AIBO [6] with reinforcement learning. By using a clicker in the similar way to a real dog training, a human can gradually train an AIBO. Dorigo also utilized shaping of operant conditioning[4]. However, a main issue of these researchs are not concerned with adaptation of a human trainer to a robot during training, and they assumed a human trainer to be well trained. Our research objective is to facilitate mutual adaptation, not to implement an interactive learning pet robot which can learn to achieve complicated tasks.

\section{AIBO: pet robot}

$\mathrm{AIBO}[2]$ is a popular pet robot having appearance like a dog. It has tactile sensors, a camera, a wireless LAN unit and so on. Fig.1 and Fig.2 show front view and rear view of AIBO ERS-7 respectively, and the positions of sensors, indicators attached on AIBO. The detail specification is shown in Table 1.

We employ OPEN-R SDK[1] programming environment for implementing sensing and executing actions on AIBO, and Ruby language to control an AIBO on a $\mathrm{PC}$ by remote brain approach.

\section{Task: easy operation of AIBO}

In this study, a task of a human and a pet robot is that a human operates an AIBO by using only three buttons(tactile back sensors in Fig.2) on the back to achieve a simple task that going forward a little way, sitting down there and going backward around a start position. Because no action is assigned to the three buttons at the beginning, a human needs to train an AIBO to execute actions by the three buttons, and this is human's adaptation to a robot. In contrast,

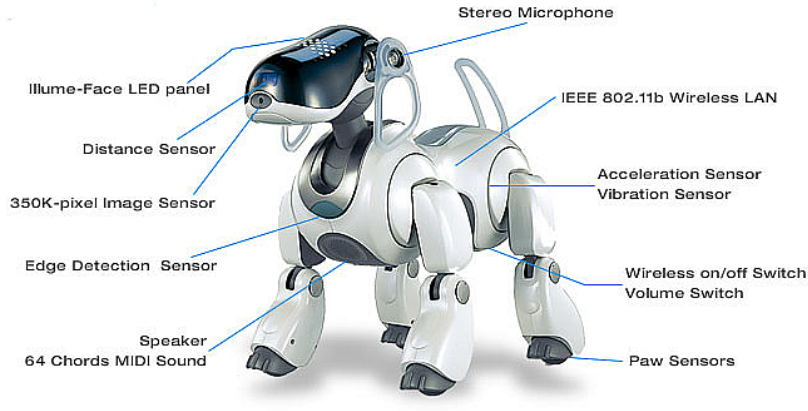

Figure 1 Sensors and indicators in front of an AIBO

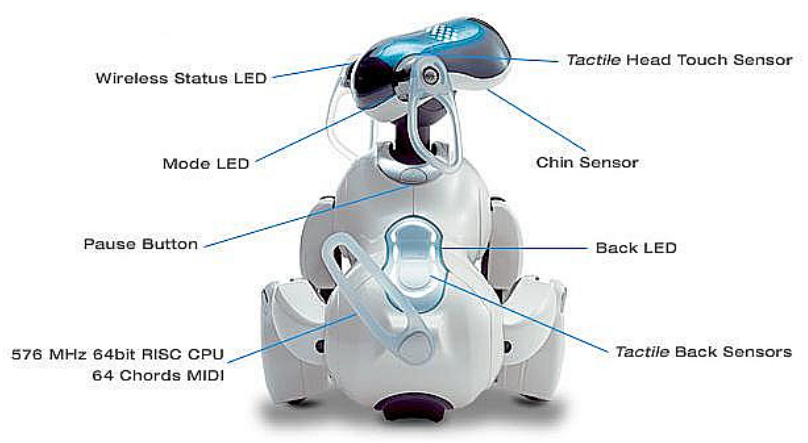

Figure 2 Sensors and indicators in back of an AIBO

an AIBO should learn to interpret commands from a human using the three buttons and execute adequate actions, and this is robot's adaptation to a human. Thus there occurs mutual adaptation between a human and a robot, and our research objective is to facilitate the mutual adaptation. We try to accelerate human's adaptation to a robot by designing suitable robot's behavior for a dog-type pet robot.

\section{Robot's adaptation to a human: be- havior learning}

In order to achieve the task mentioned before, we utilize classical conditioning as learning algorithm of AIBO. Though there are a lot of machine leaning algorithm like reinforcement learning, GA, GP, we consider classical conditioning is suitable for our study because it is a simple learning algorithm based on animal behavior to achieve our task efficiently. Reinforcement learning is also based on operant conditioning of animals, however the learning needs a large number of trial and error to converges to the optimal policy and human subjects can not bear such heavy teaching. Note that our research objective is to develop a method to facilitate mutual adaptation, not to build 


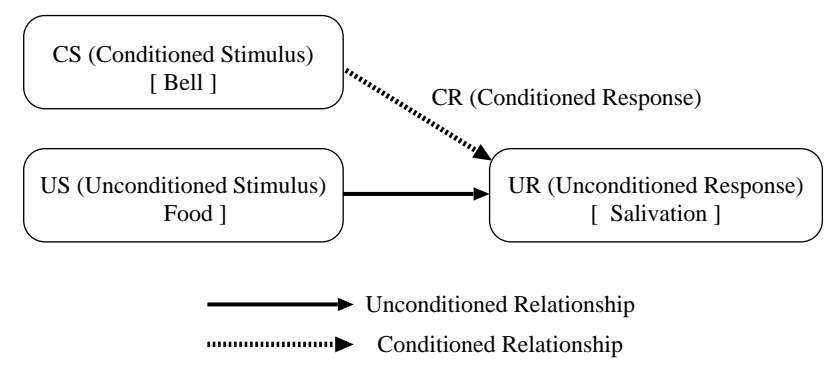

Figure 3 Classical conditioning

a robot to learn many tasks.

\subsection{Classical conditioning}

Classical conditioning[5] is well known learning of an animal as experiments of Pavlov's dog. Fig. 3 shows a framework of classical conditioning. At the beginning, a dog does not respond to ringing bell(CS: Conditioned Stimulus), and salivates(UR: Unconditioned Response) responding to food(US: Unconditioned Stimulus) in front of it. After ringing bell and food were given to the dog several times, CS and US are associated and the dog eventually salivates by only ringing bell, not food. This is a process of classical conditioning.

Thought tasks that classical conditioning achieves is more restricted than reinforcement learning, it has a simple procedure, quick convergence of learning and sufficient learning ability to achieve the task in our framework. Thus we utilize classical conditioning as behavior learning of a pet robot.

\subsection{Computational model}

Various computational models have been proposed and their ability are experimentally investigated[3]. We utilize a Klopf model[7] as classical conditioning of a pet robot because it has simple structure and one of the best models to describe classical conditioning phenomena of an animal[3].

Fig.4 shows a neuron model of Klopf. A neuron has multiple inputs from other neurons and a single output to other neurons. Each input has weight to be modified by learning and the output value(activity) is determined by a function of the inputs. The following formula show the output function (1) and the learning procedure (2)-(6) and of a neuron $j$, where $s_{i}$ is a sensed data, $m_{j}(t)$ is activity, $c_{j}$ is a learning rate to adjust the degree of updating weights and $\tau$ controls how many past data the update is computed with. The parameters $\tau$ and $c_{k}$ are determined experimentally. We slightly modified the original Klopf learning equations so that learning can be done more

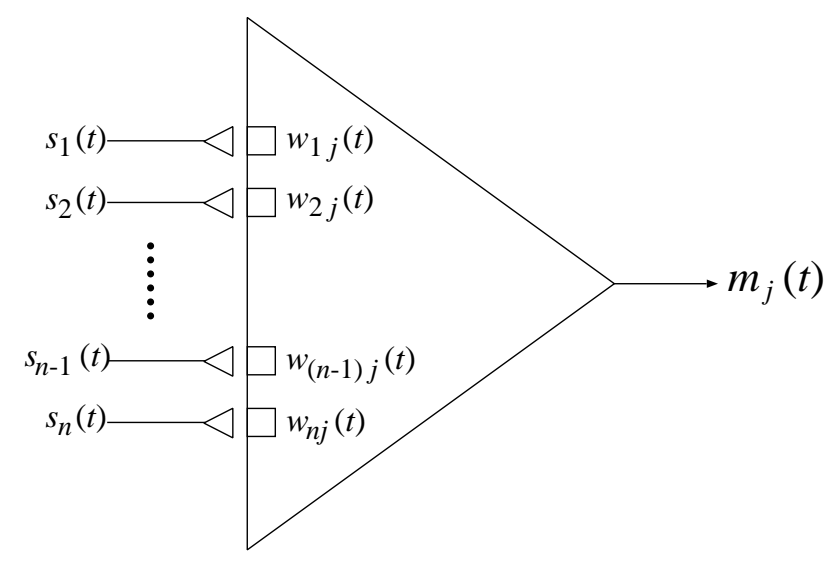

Figure 4 Klopf neuron model

sensitively to input changes and more efficiently.

We assign a neuron to an action, and sensor data are inputed to each neuron and the output of a neuron is activity. Fig.5 shows the whole neural network used for action selection of an AIBO indicating sensed data and actions to be mentioned later. The weights of inputs for US are fixed, and those for CS are updated by formula (2)-(6) every action execution. The AS unit is a function which outputs the action with the maximum activity only when the activity is more than activity threshold $\theta$. A cycle of sensing, action selection, action execution and learning is repeated.

$$
\begin{aligned}
m_{j}(t) & =\sum_{i=1}^{n} w_{i j}(t) s_{i}(t) \\
w_{i j}(t) & =w_{i j}(t-1)+\Delta w_{i j}(t) \\
\Delta w_{i j}(t) & =\Delta m_{j}(t) \sum_{k=1}^{\tau} c_{k}\left|w_{i j}(t-k)\right| \Delta s_{i}(t-k)(3) \\
\Delta w_{i j}(t) & =w_{i j}(t+1)-w_{i j}(t) \\
\Delta m_{j}(t) & =m_{j}(t)-m_{j}(t-1) \\
\Delta s_{i}(t-k) & =s_{i}(t-k)-s_{i}(t-k-1)
\end{aligned}
$$

\subsection{Sensors and motor commands}

Since the task $\S 3$ needs three actions; go forward, go backward and sit down, we use three motor commands corresponding to the three actions for AIBO and no other redundant one is not employed. Sensor data we used are in the following.

- Pressing the head sensed by a tactile sensor on the head.

- Pressing the back sensed by tactile sensors(buttons on the back) on the back. 


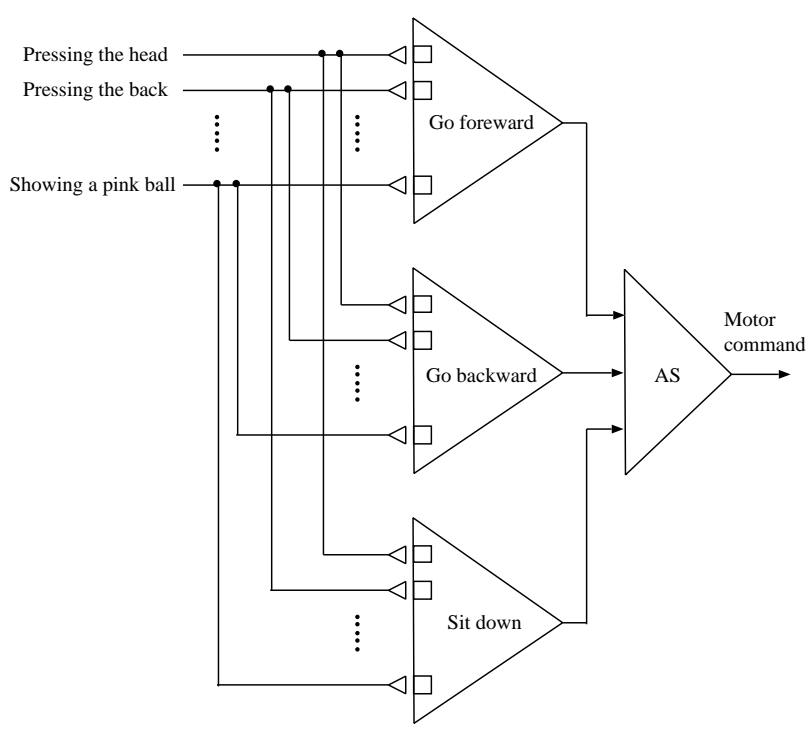

Figure 5 Action selection of our AIBO

- Pressing the chest sensed by an edge dectection sensor on the chest.

- Pressing the chin sensed by a touch sensor on the chin.

- Showing a pink ball sensed by a CMOS camera image sensor on the top of a nose.

\section{Interaction design for facilitating adaptation}

We identify the human's adaptation in mutual adaptation of our framework and develop a method to facilitate the human's adaptation by using like-dog heuristics.

\subsection{Human's adaptation to a robot}

In our framework, the adaptation of a pet robot is just behavior learning by classical conditioning, and the human's adaptation is mainly consisting of the following searches because a human does not know US and CS at the beginning.

- Searching US: A human need to search US by trial and error.

- Searching CS: A human need to search CS by trial and error in the similar to unconditioned.

However, searching CS is not necessary because we tell a human to use three buttons on the AIBO's back as CS. Thus we attempt to facilitate human's searching US.

\subsection{Facilitating adaptation with like-dog heuristics}

We propose like-dog heuristics to facilitate mutual adaptation between a human and a dog-type pet robot. This heuristics means that when a human has interaction with a dog-type pet robot, he/she inevitably models it as a dog and he/she behaves to it in the similar way to interact with a dog. This like-dog heuristics provides us the policy of designing interaction between a human and a dog-type pet robot. In this study, we can apply like-dog heuristics to AIBO's behavior for US/UR.

As mentioned earlier, a human needs to search US by trial and error because he/she does not know which stimulus is US at the beginning. In order to facilitate this search, we can apply like-dog heuristics by designing AIBO's behavior for US/UR similarly to a dog. By like-dog heuristics, we can expect that a human is likely to find the similar US to real dog's ones. Thus, by designing AIBO's behavior for US like a real dog's ones, we can facilitate human's adaptation to an AIBO.

Hence we design natural behavior of an AIBO for US/UR in the following, and they are used in experiments.

- If an AIBO finds a pink ball, it'll go forward.

- If a human presses the back of an AIBO, it'll sit down.

- If a human presses the chest of an AIBO, it'll go backward.

These behavior are considered to be natural as a dog's one. By designing such dog-like behavior for US, we expect to facilitate the human's search for US and classical conditioning. The effort of this interaction design will be evaluated experimentally in the next section.

\section{Experiments}

We conduct preliminary experiments for evaluating the like-dog heuristics and verifying feasibility of training an AIBO by classical conditioning.

To evaluate like-dog heuristics, we compare efficiency of finding US with or without like-dog heuristics. Meanwhile, we investigate a human can actually train an AIBO by classical conditioning for its feasibility.

\subsection{Experimental setting}

We used four subjects(male graduate students in a computer science department) and an AIBO ERS7 on which classical conditioning was implemented in the way of $\S 4$ for all experiments. 

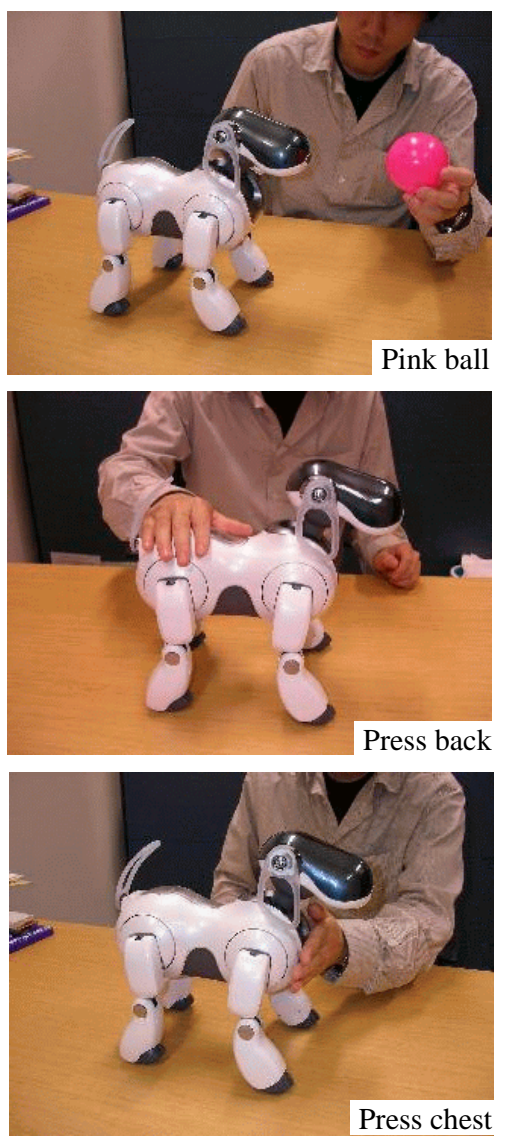

Figure 6 Dog-like US

To compare with natural behavior for US/UR with like-dog heuristics in $\$ 5.2$, we use unnatural behavior for US/UR in the following. The first and third behavior have opposite actions to natural ones. Note that the condition of the second behavior, press the head, is unnatural though it is easily satisfied only by head touch.

- If an AIBO finds a pink ball, it'll go backward.

- If a human presses the head of an AIBO, it'll sit down.

- If a human presses the chest of an AIBO, it'll go forward.

The experiments consist of two phases like the following.

\subsubsection{Finding US}

Before starting experiments, we give subjects the following instructions.

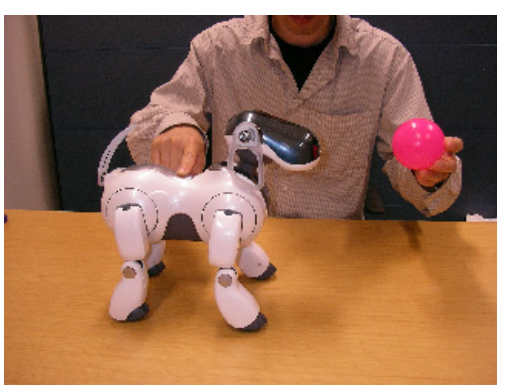

Figure 7 Classical conditioning for a pink ball

- An AIBO has three actions, go forward, go backward and sit down which can be executed by certain stimulus.

- Please find the three stimulus to execute actions by touching it, showing it something and so on.

- An AIBO likes a pink ball.

After the instructions, six behavior consisting of three natural and three unnatural behavior are in implemented on an AIBO one by one in random ordering for each subject, and they try to find the stimulus of behavior one by one. We shoot a video and investigate the time for a subject to find each stimulus.

\subsubsection{Classical conditioning}

After the first phase, we ask the same subject to train an AIBO by classical conditioning, and the following are instructions.

- We explain principle and procedure of classical conditioning as described in $§ 4.1$.

- We explain a task as described in $\S 3$.

For each subject, we implement a set of three natural behavior and a set of three unnatural behavior on an AIBO, and an subject trains an AIBO twice with different AIBO's behavior sets. Because the three buttons on the back are used to CS, we modified the condition of natural behavior for sitting down into touching the chin. We shoot a video and investigate the time for a subject to finish learning to achieve the task. Fig.7 shows a snapshot in which an subject trains an AIBO with a pink ball. From this figure, you can see he simultaneously gives CS(pushing a button in the back) and US(showing a pink ball) to an AIBO.

\subsection{Experimental results and discussion}

Fig. 8 shows experimental results of the first phase to find US. The average time to find US over subjects 


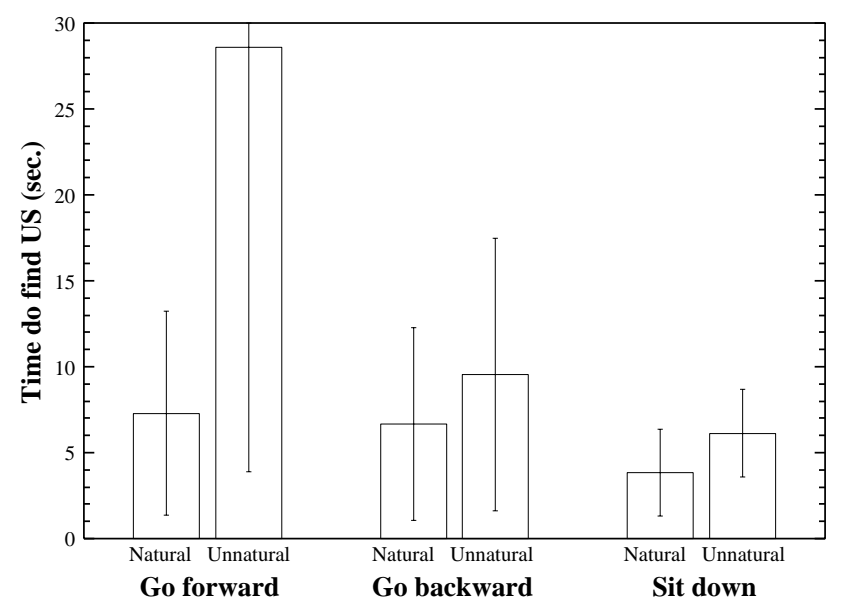

Figure 8 Experimental results of finding US

and the standard deviation are indicated. Seeing from this graph, the time to find stimulus by the natural behavior is shorter than that by the unnatural behavior for all three actions. Hence, though the sample number is small and statistic test is not available, we almost conclude the natural behavior makes a human to find US more efficiently than the unnatural behavior and like-dog heuristics is promising to facilitate human's adaptation to an AIBO in our framework. Currently we are trying to conduct more systematic experiments with more subjects.

Table 2 shows average and the standard deviation in parentheses of the time to finish learning. The training of all subjects finished about four minutes and these facts support the feasibility of classical conditioning to train a pet robot for a simple task. The subjects understood AIBO's US through the previous experiments, and this might training more efficient. Training with unnatural behavior slightly outperforms one with natural behavior, however a subject with unnatural behavior misled an AIBO by confusion caused from the unnatural behavior.

Through the experiments, we found out the familiarity of a subject to a real dog, a pet robot, might have significant influence on whether he/she feels a given behavior natural or not. Thus, in order to obtain precise results, subjects should be controlled and grouped or the results are normalized depending on such familiarity.

\section{Conclusion}

We proposed and implemented a mutual adaptation framework of a human and a pet robot, and developed like-dog heuristics to facilitate the human's adaptation
Table 2 Experimental results of classical conditioning: Average time(sec.)

\begin{tabular}{|c|c|}
\hline Natural behavior & Unnatural behavior \\
\hline $198.5(40.88)$ & $177.75(28.34)$ \\
\hline
\end{tabular}

to a pet robot. In our framework, a pet robot does behavior learning and a human learns how to train it. A pet robot utilizes classical conditioning, and we proposed like-dog heuristics to facilitate human's adaptation to a pet robot. Finally we conducted preliminary experiments using an AIBO to evaluate the heuristics, and verified effectiveness of like-dog heuristics.

The experimental results were obtained with a small number of subjects. Thus we will conduct more systematic experiments with more subjects, and verify the effectiveness of like-dog heuristics. Furthermore, we have a plan to develop various interaction designs using like-dog heuristics and evaluate them experimentally.

\section{References}

[1] Aibo sde web site. http://openr.aibo.com/.

[2] Aibo web site. http://www.sony.net/Products/aibo/.

[3] C. Balkenius and J. Morn. Computational models of classical conditioning: a comparative study. In Proceedings of the Fifth International Conference on Simulation of Adaptive Behavior, 1998.

[4] M. Dorigo and M. Colombetti. Robot Shaping: An Experiment in Behavior Engineering. MIT Press/Bradford Books, 1998.

[5] W. Hill. Learning: A survey of psychological interpretations. Harper and Row, 1985.

[6] F. Kaplan, P.-Y. Oudeyer, E. Kubinyi, and A. Miklosi. Robotic clicker training. Robotics and Autonomous Systems, 38(3-4):197-206, 2002.

[7] A. H. Klopf. A neuronal model of classical conditioning. Psychobiology, 16(2):85-123, 1988.

[8] T. Komatsu, K. Suzuki, K. Ueda, K. Hiraki, and N. Oka. Mutual adaptive meaning acquisition by paralanguage information: Experimental analysis of communication establishing process. In The Proceedings of the 24th Annual Meeting of the Cognitive Science Society, pages 548-553, 2002.

[9] T. Ono and M. Imai. Reading a robot's mind: A model of utterance understanding based on the theory of mind mechanism. In Proceedings of the Seventeenth National Conference on Artificial Intelligence, pages 142-148, 2000.

[10] S. Yamada and T. Yamaguchi. Mutual adaptation to mind mapping in human-agent interaction. In IEEE International Workshop on Robot-Human Interaction, pages 105-110, 2002. 